\title{
COMPORTAMENTO DO ALGODOEIRO HERBÁCEO \\ (Gossypium hirsutum latifolium Hutch.) \\ E CON TROLE DE PLANTAS DANINHAS COM O USO DOS HERBICIDAS DIURON E SETHOXYDIM
}

\author{
N.E. DE M. BELTRÃO**, J.F. DA SILVA***, A.J. DA SILVEIRA***, C.S. SEDYAMA***, L.M. \\ DA COSTA $* * * \&$ M.A. OLIVA*** \\ * Parte da tese do primeiro autor para obten - \\ ção do grau de "Doctor Scientiae" na Uni - \\ versidade Federal de Viçosa, MG. \\ ** Pesquisador da EMBR APA/CNP - Algodão, \\ Cx. Postal 174 - 58.100 - Campina Grande, \\ PB. \\ -* Professor da Universidade Federal de Viço - \\ sa. 36.570 - Viçosa, MG.
}

\section{RESUMO}

Com a finalidade de verificar o comportamento do algodoeiro herbáceo, cultivar IAC-17, bem como o controle de plantas daninhas e aspectos competitivos do complexo floristico infestante sobre a cultura, na presença dos herbicidas diuron e sethoxydim, foi realizado um ensaio no município de Viçosa, Minas Gerais. O solo do local experimental, Podzólico Vermelho-Amarelo, apresenta textura argilosa, com $1,38 \%$ de carbono orgânico e de baixa fertilidade natural.

$O$ diuron foi aplicado em pré-emergência nas doses de 0,$0 ; 0,8 ; 1,6$ e 2,4 kg/ha e o sethoxydim, em pós-emergência, nas doses de 0,150, 300, 450 e $600 \mathrm{~g} / \mathrm{ha}$.

O ens aio foi instalado em blocos ao acaso, com 21 tratamentos em esquema fatorial $(4 \times 5+1)$, sendo 20 deles envolvendo o controle químico, resultantes de todas as combinações das doses desses herbicidas e uma testemunha relativa onde o controle foi realizado com o uso da enxada.

Avaliaram-se várias características do crescimento e desenvolvimento da cultura, tais como área foliar, índice de área foliar, rendimento de algodão em rama, altura da planta, diâmetro do caule etc.; e, por meio de métodos sinecológicos, a densidade populacional e peso da fitomassa hidratada epígea das espécies daninhas dominantes, e o total de todas as espécies.

O diuron exerceu um elevado controle de latifoliadas, como botão-de-ouro (Galinsoga parviflora Cav.) e picão-preto (Bidens pilosa L.), nas doses de 1,6 e $2,4 \mathrm{~kg} / \mathrm{ha}$. O sethoxydim mesmo na menor dose testada $(150 \mathrm{~g} / \mathrm{ha})$ controlou totalmente o capim-marmelada (Brachiaria plantaginea (Link.) Hitch). Nenhum dos herbi- cidas controlou a falsa-serralha (Emilia sonchifolia $D C$.), porém referida planta daninha não reduziu o crescimento da cultura, mostrando-se de baixa força de competição. As plantas daninhas que apresentaram maiores forças de competição foram o botão-de-ouro, por apresentar maior densidade populacional, e o capim-marmelada, por ser de maior agressividade.

Palavras chave: Algodoeiro, herbáceo, plantas daninhas, diuron, sethoxydim, competição.

\section{SUMMARY}

THE BEHAVIOR OF UPLAND-TYPE COTTON (G. hirsutum latifolium Hutch.) AND THE CONTROL OF WEEDS AFTER THE USE OF DIU RON AND SETHOXYDIM HERBICIDES.

To verify the behavior of the c. IAC-17, as well as, the control of weeds and competitive aspects of the infesting floristic complexes over the cotton culture under the presence of the herbicides, diuron and se thoxydium, a trial was contucted in Viçosa, Minas Gerais.

The soil at the experimental site, Podzolic Red-yellow, had a clay texture with $1,38 \%$ of organic carbon an low natural fertility.

Diuron was applied at pre-emergence time at the rates of 0,$0 ; 0,8 ; 1,6$ and $2,4 \mathrm{~kg}$ a.i./ha and sethoxydim at post-emergence at the rates of $0,150,300,450$ and $600 \mathrm{~g}$ a.i./ha.

The trial was set up in a randomized blocks design with 21 treaments under a factorial scheme $(x 5+1)$. Out of them, 20 composed all the combinations with different dosis of the two herbicides under study plus a relative control weeded with the aind of a mattock.

Several traits concerning growth and plant 
development were evaluated, such as leaf area, leaf area index, seed-cotton yield, plant height, stem diameter. By means of synecological me thods, the population density, hydrated epigeous phytomase of dominant weed species, and the total of all species were evaluated.

Diuron exerted a high control over latifolia tes such as Galinsoga parviflora $\mathrm{Ca}^{\mathrm{v}}$. and Bidens pilosa L., at the rates of 1,6 and $2,4 \mathrm{~kg}$ a.i./ha, sethoxydim, even using the lowvest tested rate (150 g. a.i./ha) fully controled Brachiaria plantaginea (Link.) Hitch.

None of the herbicides was able to control Emilia sonchifolia DC. This species although being considered an important weed did not affect the normal crop development because of its low competition ability.

The weeds showing highest rates of competition were G. parviflora (due to high population density) and B. plantaginea, because of its greater aggresivity.

Keywords: Upland-type cotton, weeds, diuron, sethoxydim, competition.

\section{INTRODUÇÃO}

O algodœiro herbáceo (Gossypium hirsutum latifolium Hutch.), como a maioria das plantas cultivadas, é extremamente sensível à competição imposta pelas plantas daninhas. Divers os trabalhos (3, $4,5,8,22,31)$ mostram que quando não controladas estas reduzem o rendimento da cultura, além de algumas delas depreciarem a qualidade da fibra, reduzindo o tipo no processo classific atório comercial (30).

O controle da vegetação daninha e a colheita são as duas práticas que mais oneram o custo de produção da cultura do algodão, sendo que o uso racional de herbicidas, aliado a outras práticas de cultivo, poderá reduzir o custo total de produção, uma vez que, na maioria das regiões produtoras desta malvácea está havendo falta de mão-de-obra, além da constante, porém justa, elevação do preço do dia/homem.

O diuron é um dos herbicidas mais recomendad os e utilizados no controle de plantas daninhas na cultura do algodão, isolado, combinado ou misturado com outros herbicidas $(1,13,14,27,37)$, sendo mais eficaz no controle de dicotiledôneas (37), embora exerça uma forte fototoxidez em várias monocotiledôneas anuais.

Diversos graminicidas têm sido associados ao diuron visando o controle de um mai or número de espécies, redução do custo do controle, decréscimo da fitotoxidade à cultura etc., em pré-plantio incorporado ou em pré-emergência. No entanto, os produtos recomendados até o presente, para esta malvácea, em pósemergência, são na maioria de ação total, não seletivos, ou de baixa seletividade reque rendo que a apl icação seja dirigida $(14,15,17,36)$. Tal fato promove aumento do custo de aplicação, devido a demandar mais tempo na operação, eleva a possibilidade de injúria ao algodoeiro, além de requerer mão-de-obra mais especializada, uso de protetores e controle ineficiente entre as plantas da cultura, dentro do sulco de plantio. Assim, o sethoxydim por ser um herbicida seletivo para latifoliadas surge como sendo uma grande opção para o controle de gramíneas precoces ou tardias na cultura.

Os objetivos deste trabalho foram : verificar a influência dos herbicidas diuron, em pré-emergência e sethoxydim em pós-emergência em alguns atributos do crescimento e desenvolvimento do algodoeiro herbáceo ; quantificar os efeitos de tais herbicidas no controle de plantas daninhas e estimar a pressão de competição de algumas plantas daninhas que ocorrem em agroecossistem as cotonícolas.

\section{MATERIAIS E MÉTODOS}

O experimento foi instalado no dia 23 de outubro de 1981 em uma das áreas experimentais da Universidade Federal de Viçosa ( U.F.V. ). O solo foi preparado através de uma aradura e duas gradagens, 15 dias antes da data de plantio e gradea do momentos antes do plantio, sendo um Podzólico Vermelho-Amarelo e apresentou os atributos químicos e composição granulométrica mostrados no quadro 1.

A precipitação pluvial ocorrida no período de 23 dias antes do plantio à colheita, foi de $1.441,0 \mathrm{~mm}$ com maiores quedas nos meses de novembro (391,3 
$\mathrm{mm})$, janeiro $(257,0 \mathrm{~mm})$ e março $(276,9 \mathrm{~mm})$. A região de Viçosa apresenta, de acordo com os dados climáticos de 29 anos, $80 \%$ de umidade relativa média anual, temperatura minima de $14^{\circ} \mathrm{C}$ e máxima de $26^{\circ} \mathrm{C}$.

O solo foi adubado no plantio, utilizando-se 20 - 90 - $100 \mathrm{~kg} / \mathrm{ha}$ de N, P205 e $\mathrm{K} 20$, aplicado em sulco e ao lado das sementes, utilizando-se as fontes : sulfato de amônio, superfosfato simples e cloreto de potássio. Aos 50 dias do plantio, real izou-se uma adubação de cobertura com sulfato de amônio, na quantidade de $20 \mathrm{~kg} / \mathrm{ha}$ de $\mathrm{N}, 10 / 12 \mathrm{~cm}$ ao lado da fileira de plantio.

O plantio foi realizado com plantadeira de tração mecânica, regulada para uma médi a de 35 sementes por metro linear de sulco.

Utilizou-se o cultivar IAC-17, cujas sementes foram oriundas da Região do Triângulo Mineiro da safra 1980/81, de elevado valor cultural.

Quadro 1 - Análises química e granulométrica de uma amostra composta do solo do local experimental. Viçosa, Minas Gerais, 1981(1),

\begin{tabular}{|c|c|c|c|c|c|c|}
\hline \multirow{3}{*}{ Química } & \multicolumn{6}{|c|}{ Atributos } \\
\hline & \multirow{2}{*}{$\begin{array}{c}\left.\frac{\mathrm{pH}}{\left(\mathrm{em}^{\mathrm{H}_{2}}\right.}{ }^{\mathrm{O}}\right) \\
4,7\end{array}$} & \multicolumn{2}{|c|}{$\mathbf{P}_{\text {ppm }} \mathbf{K}$} & \multicolumn{2}{|c|}{$\begin{array}{c}\mathrm{Ca}++\underset{\text { eq. } \mathrm{mg} / 100 \text { cc solo }}{\mathrm{Mg}++} \mathrm{Al}+++ \\
\end{array}$} & \multirow{2}{*}{$\begin{array}{c}\begin{array}{c}\text { Carbono } \\
\text { orgânico } \\
\%\end{array} \\
1,38 \\
\end{array}$} \\
\hline & & 4 & 41 & 2,4 & 0,3 & \\
\hline \multicolumn{7}{|c|}{ Análise Granulométrica } \\
\hline Separatas & & \multicolumn{3}{|c|}{ Percentagem } & \multicolumn{2}{|c|}{ Classificação Textural } \\
\hline Areia grossa & & \multicolumn{3}{|c|}{26} & & \multirow{3}{*}{$\cdot$} \\
\hline Areia fina & & & & & & \\
\hline Silte & & & & & & \\
\hline Argila & & & & & & \\
\hline
\end{tabular}

(1) Análises realizadas pelos laboratórios de Química e Física do Solo da Universidade Federal de Viçosa.

o herbicida diuron foi aplicado 24 horas após o plantio, em solo úmido, utilizando-se de pulverizador costal manual. calibrado para uma vazão de 350 1/ha da calda. O herbicida sethoxydim foi aplicado em pós emergência total, aos 37 dias do plantio, gastando-se 400 1/ha da calda.

Utilizou-se o delineamento experimental de blocos ao acaso com três repetições e 21 tratamentos. Os tratamentos herbicídicos foram todos combinações de quatro doses de diuron $\left(0,0 ; 0,8^{3} ; 1,6 \mathrm{e}\right.$ $2,4 \mathrm{~kg} / \mathrm{ha}$ ) e cinco doses de sethoxydim 0 ; $150 ; 300 ; 450$ e $600 \mathrm{~g} / \mathrm{ha}$ ), constituin do um fatorial $4 \times 5$, mais um tratamento adicional, cujo controle de plantas daninhas foi feito com utilização de enxada.
Foram realizadas quatro capinas no tratamento com o controle mecânico ma nual e aos 85 dias do plantio, época em que as plantas daninhas já não competem mais com a cultura $(4,5,22,35)$, realizouse uma limpeza geral à enxada em todo ensaio com a finalidade exclusiva de facilitar a colheita.

As unidades experimentais tiveram uma área de $20 \mathrm{~m}^{2}(4 \mathrm{~m} \times 5 \mathrm{~m})$, sendo útil a área central de $10 \mathrm{~m}^{2}$.

A vegetação daninha da área experi mental era constituída pelas seguin te: espécies : falsa-serralha (Emilia sonchifolia D.C.), serralha-mans a (Sonchus oleraceous L.), capim-marmelada (Brachiaria plantagi- 
nea (Link) Hitch.), picão-amarelo (Galin-soga parviflora Cav. ), que eram as dominantes. As demais foram: picão-preto (Bidens pilosa L.), corda-de-viola (Ipomoea sp.), amendoimbravo (Euphorbia prunifolia Jacq.), trapoeraba (Commelina sp.) e trevo (Oxalis sp.), identificadas segundo as informações de Cárdenas et al. (12) e Brandão et al. (6) .

A fitotoxicidade foi avaliada pela escala EWRC (European Weed Research Council, 20 dias após a aplicação de cada herbicida.

Avaliaram-se as seguintes variáveis relaciona das com a cultura : lotação inicial e final, diâmetro do caule a dois centímetros do colo e altura da planta aos 90 dias do plantio e na colheita, números de ramos por planta na colheita, rendimento do algodão em rama, número de folhas, área foliar por planta e índice de área foliar (IAF) aos 90 dias da colheita.

Para altura da planta, diâmetro do caule e variáveis relacionadas com a folhagem da planta, as medições foram realizadas numa amostra por unidade experimental, de cinco plantas. A área foliar foi determin ada pelo método de Ashley et al. (2) e o IAF, segundo as informações de Hunt (21).

Para verificação dos efeitos dos herbicidas sobre a comunidade infestante, utilizaram-se métodos sinecológicos, envolvendo parâmetros de densidades populacionais e pesos das fitomassas hidratad as epígeas $(7,19)$, aos 60 dias do plantio. As avaliações foram realizad as por espécie e analisadas apenas as dominantes, e o total de todas as espécies presentes.

As anál ises estatísticas foram feitas pelos métodos convencionais, citados por La Loma (25) e Pimentel Gomes (29) e como teste de comparação de médias, usou-se o de Tukey ao nível de 5\% de probabilidade. Quando necessário, os dados foram transformados para $\mathrm{x}+1$ ou $\log$ $(x+1)$ de acordo com as informações de Calzada Benza (11).

\section{RESULTADOS E DISCUSSÃO}

O resumo das análises de variância dos dados referentes a rendimento de algodão em rama, diâmetro do caule e altura da planta, aos 90 dias do plantio e na colheita, número de ram os veg etativos na colheita e lotação de plantas após o desbaste e na col heita, enc on tra-se no quadro 2. Verifica-se que ocorrer am efeitos significativ os para o diuron em todas as variáveis e do sethoxydim apenas para rendimento e lotação de plantas.

A significância estatística verificada entre o fat orial (tratam ent os her bic ídi cos) e a testemunha ( controle mecânico), denota, "a prio ri", supe rioridade do último. Realmente, ele apresentou valores superiores para todas as variáveis retro mencionadas. No entanto, a aplicação do teste de Tukey às médias dos tratamentos, revelou que algumas das combin ações herbicídicas, especialmente quando o diuron foi utilizado nas doses mais elevadas (1,6 e 2,4 kg/ha ), não foram diferentes do controle mecânico. Deste modo, a inferioridade apre sentada pelo fatorial é devido ao efe ito méd io dos tratamentos, incluída a testemunha absolutá, onde não se aplicou herbicidas.

No quadro 3 encontram-se as médias dos trata men tos herbi cíd icos, con siderando o rendimento de algodão em rama. Verifica-se que a competição imposta pelas plantas daninhas, tratamento sem uso de herbicidas, reduziu drasticamente tal variável, mostrando que o algodoeiro, como a maioria das espécies cultivadas é bastante sensível ao processo emulativo promovido pelo complexo florístico daninho. Os resultados, assim, estão de acordo com a maioria das pesquisas anteriores $(3,4,5,8,22,31)$.

$\mathrm{O}$ algodoeiro, quando realizou-se $\mathrm{o}$ controle mecânico, apresentou um rendimento médio de $1.666,00 \mathrm{~kg} / \mathrm{ha}$ de algo dão em rama.

Verifica-se, pelo quadro 3, que o diuron à medida que a dose foi aumentada, o rendimento elevou-se. $\mathrm{O}$ mesmo fato ocorreu com o sethoxydim, embora com 
Quadro 2 - Resumo das análises de variância dos dados de rendimento de algodão em rama, em $\mathrm{kg} / \mathrm{ha}$, diâmetro caulinar aos 90 dias do plantio e na colheita, em $\mathrm{cm}$, altura plantular aos 90 dias do plantio e na colheita, em $\mathrm{cm}$, número de ramos vegetativos por planta (RM) na colheita, e lotação de plantas em relaçåo à populaçăo máxima, após o desbaste e na colheita, em percentagem, em função dos tratamentos herbicídicos (fatorial) e o mecânico (testemunha relativa). Viçosa, Minas Gerais, 1982.

\begin{tabular}{|c|c|c|c|c|c|c|c|c|c|}
\hline \multirow[b]{2}{*}{ Fonte de Variação } & \multirow{2}{*}{$\begin{array}{c}\text { Grau de } \\
\text { Liberdade }\end{array}$} & \multicolumn{8}{|c|}{ QUADRADO MEDIO } \\
\hline & & Rendimento & $\begin{array}{c}\text { Diâmetro } \\
90 \text { dias }\end{array}$ & $\begin{array}{l}\text { Diâmetro } \\
\text { Colheita }\end{array}$ & $\begin{array}{l}\text { Altura } \\
90 \text { dias }\end{array}$ & $\begin{array}{c}\text { Altura } \\
\text { Colheita }\end{array}$ & $\mathbf{R M}$ & $\begin{array}{c}\text { Lotação após } \\
\text { Desbaste }\end{array}$ & $\begin{array}{c}\text { Lotaçâo na } \\
\text { Colheita } \\
\end{array}$ \\
\hline Blocos & 2 & $762830,000^{* *}$ & $0,375^{\star *}$ & $0,151^{\star *}$ & $1274,714^{* \star}$ & $3289,906^{* *}$ & 0,173 & $59,663^{*}$ & $179,763^{\star}$ \\
\hline Fatorial & (19) & $501351,742^{\star \star}$ & $0,204 * *$ & $0,072^{* *}$ & $1065,294 * *$ & $836,874^{* *}$ & $0,235^{*}$ & $176,242^{* *}$ & $287,207^{\star \star}$ \\
\hline Diuron (D) & 3 & $2722707,000^{* *}$ & $1,216^{ \pm *}$ & $0,371^{* *}$ & $6493,438^{* \hbar}$ & $3754,940^{* *}$ & $0,725^{* *}$ & $347,635^{* *}$ & $794,225^{* *}$ \\
\hline Sethoxydim (S) & 4 & $233856,875^{\text {** }}$ & 0,020 & 0,031 & 52,542 & 122,508 & 0,082 & $215,234^{* *}$ & $239,816^{* k}$ \\
\hline D X s & 12 & 35177,805 & 0,600 & 0,011 & 45,842 & 345,479 & 0,164 & $120,397 * *$ & $176,250^{ \pm \star}$ \\
\hline Fatorial vs Test. Relativa & 1 & $2802739,313^{* *}$ & $0,967^{\star \star}$ & $0,508^{* *}$ & $5980,187^{* *}$ & $2792,375^{\star \star *}$ & $6,004^{* *}$ & 11,804 & 31,928 \\
\hline Tratamentos & 20 & $616421,063^{\text {t** }}$ & $0,242^{* *}$ & $0,094^{* *}$ & $1311,038^{* *}$ & $934,649^{\text {** }}$ & 0,523 ** & $168,021^{* *}$ & $274,443 * *$ \\
\hline Residuo & 40 & 52402,598 & 0,020 & 0,024 & 71,829 & 297,684 & 0,125 & 16,300 & 51,157 \\
\hline CV (\%) & & 31,67 & 18,96 & 13,55 & 14,59 & 17,77 & 105,14 & 4,18 & 7,56 \\
\hline
\end{tabular}

* Significativo ao nivel de $5 \%$ de probabilidade pelo teste $\mathrm{F}$.

** Significativo ao nível de $1 \%$ de probabilidade pelo teste F. 
acréscimos menores. Isto provavelmente foi devido ao fato de que a maioria das plantas daninhas do agroecos sistema era de dicotiledôneas anuais controladas pelo diuron.

Os dados submetidos à análise de regressão, indicaram uma resposta do rendime nto da natur eza line ar múltipla (fi gura 1), sendo função das doses de ambos os produtos testados, embora 0 coeficiente de regressão para dose s do diuron tenha sido, em termos relativos, superior ao do sethoxydim.

A competição reduziu o diâmetro caulinar, altura plantular e número de ramos vegetativos por planta (quadro 4 ). Resultados semelhantes foram obtidos por Beltrão et al (9) e Robinson (31), este último, com referência especial à altura do algodoeiro.

Tais resultados mostram que a competição promove alterações aposimplásticas irreversíveis no algodoeiro.

Para a maioria das variáveis que compõem o quadro 4, uma dose de 1,6 $\mathrm{kg} / \mathrm{ha}$ de diuron, independente da dose utilizada do sethoxydim, já foi suficiente para a promoção do crescimento da cultura, por livrá-la da competição das latifoliadas e também, pelo fato, de que o diuron, embora não tenha exercido um bom controle da $B$. plantaginea, reduziu bastante sua agressividade. Por outro lado, o sethoxydim controlou bem essa gramínea, mas, sem o diuron, as de-

Quadro 3 - Médias do rendimento do algodão em rama, $\mathrm{kg} / \mathrm{ha}$, em função das doses de diuron e sethoxydim (kg/ha). Viçosa, Minas Gerais, 1982 (1).

\begin{tabular}{|c|c|c|c|c|c|c|}
\hline \multirow{2}{*}{ Doses } & \multirow{2}{*}{ do Diuron } & \multicolumn{5}{|c|}{ Doses do Sethoxydim } \\
\hline & & 0,0 & 150,0 & 300,0 & 450,0 & 600,0 \\
\hline & 0,0 & 112,800 a A & 114,000 a A & 174,667 a A & 85,333 a A & 151,200 a B \\
\hline & 0,8 & 271,567 a AB & $464,667 \mathrm{ab} A B$ & $605,333 \mathrm{ab} A B$ & $609,999 \mathrm{ab}$ B & 859,200 b B \\
\hline & 1,6 & 678,800 a $\quad B C$ & $814,000 \mathrm{ab} \quad \mathrm{BC}$ & $888,800 \mathrm{ab}$ BC & $1042,800 \mathrm{ab} B C$ & 1206,800 b B \\
\hline 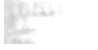 & 2,4 & 846,000 a & $1138,333 \mathrm{a}$ & 1029,200 a & $1202,800 \mathrm{a}$ & 1214,400 a $B$ \\
\hline
\end{tabular}

(1) Médias seguidas de mesma letra minúscula, nas linhas, e mai úscula, nas colunas, não diferem estatisticamente entre si, pelo teste Tukey, ao nível de 5\% de probabilidade.

Quadao 4 - Médias do diâmetro caulinar, em cm, da altura plantular, em cm, ambos aos 90 dias do plantio e na colheita e do número de ramos vegetativos por planta (RM) na colheita, em função das doses de diuron $(\mathrm{kg} / \mathrm{ha})$. Ensaio envolvendo doses de diuron e setho xydim. Viçosa, Minas Gerais, 1982 (1).

\begin{tabular}{|c|c|c|c|c|c|c|c|c|c|}
\hline \multirow{2}{*}{$\begin{array}{l}\text { DOSES DO } \\
\text { DIURON }\end{array}$} & \multicolumn{9}{|c|}{ MED I A S } \\
\hline & \multicolumn{2}{|c|}{$\begin{array}{l}\text { Diâmetro: } \\
90 \text { Dias }\end{array}$} & \multicolumn{2}{|c|}{$\begin{array}{l}\text { Diâmetro } \\
\text { Colheita }\end{array}$} & \multicolumn{2}{|c|}{$\begin{array}{l}\text { Altura: } \\
\text { 90 Dias }\end{array}$} & \multicolumn{2}{|c|}{$\begin{array}{c}\text { Altura } \\
\text { Colheita }\end{array}$} & $\mathrm{RM}$ \\
\hline 0,0 & \multicolumn{2}{|c|}{$0,333 \mathrm{~A}$} & \multicolumn{2}{|c|}{$0,900 \mathrm{~A}$} & \multicolumn{2}{|c|}{$28,200 \mathrm{~A}$} & \multicolumn{2}{|c|}{$73,413 \mathrm{~A}$} & $0,000 \mathrm{~A}$ \\
\hline 0,8 & 0,689 & B & 1,159 & B & 53,133 & B & 100,327 & B & $0,160 \mathrm{AB}$ \\
\hline 1,6 & 0,863 & $\mathrm{C}$ & 1,199 & $\mathbf{B}$ & 65,800 & $\mathrm{C}_{\mathrm{\gamma}}$ & 97,873 & B & $0,387 \quad \mathrm{E}$ \\
\hline 2,4 & 0,988 & C & 1,256 & B & 76,533 & D & 110,807 & B & 0,487 \\
\hline
\end{tabular}

(I) Em cada coluna, médias seguidas pela mesma letra não diferem entre si, pelo teste Tukey, ao nível de $5 \%$ de probabilidade. 
mais plantas daninhas dominaram o algodoeiro.

Com relação à lotação de plantas, os quadros 5 e 6 mostram que a competição reduziu bastan te o número de indivíduos da cultura, fato já observado por outros autores $(5,22,32)$. No entanto, quan do se aplicou um dos herbicidas, mesmo nas menores doses, isolad os ou combinados, tal efeito não ocorreu, devido a redução do processo competitivo global.

Algumas diferenças significativas mostradas nos quadros 5 e 6 , exce to para a testemunha absoluta (ausência de herbicidas) foram devido ao ataque, nos primeiros dias da cultura, da saúva, a qual reduziu o número de plantas em algumas parcelas.

No quadro 7 encontra-se o resumo das análi ses de variância dos dados para as variáveis referentes à folhagem do algodoeiro.

Observa-se que somente para o fator diuron é que ocorreram diferenças significativas entre os tratamentos herbici dicos.

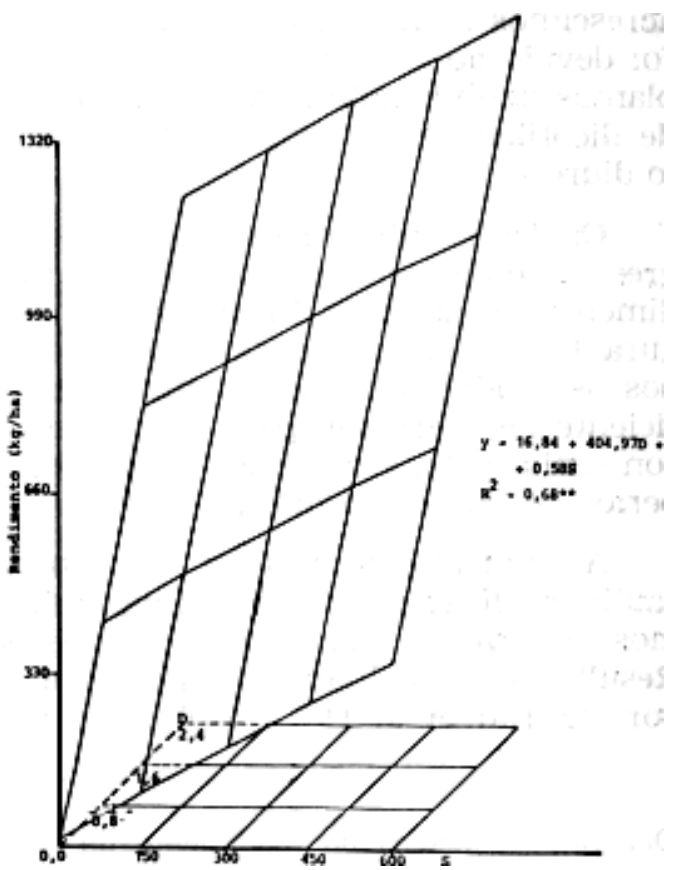

Figura 1. Representação espacial entre o rendimento de algodão em rama, em $\mathrm{kg} / \mathrm{ha}$, com doses de diuron (D), em $\mathrm{kg} / \mathrm{ha}$, e doses de sethoxydim (S), em g/ha. Viçosa, Minas Gerais, 1982.

Quadro 5 - Médias da lotação de plantas, após desbaste, em percentagem da população máxima, em função das doses de diu ron $(\mathrm{kg} / \mathrm{ha})$ e sethoxydim $(\mathrm{g} / \mathrm{ha})$. Ensaio envolvendo doses de diuron e sethoxydim. Viçosa, Minas Gerais, 1982 (1).

\begin{tabular}{|c|c|c|c|c|c|}
\hline \multirow{2}{*}{$\begin{array}{c}\text { DOSES DO } \\
\text { DIURON }\end{array}$} & \multicolumn{5}{|c|}{ DUSES DE SETHUXYDIM } \\
\hline & 0,0 & 150,0 & 300,0 & 450,0 & 600,0 \\
\hline 0,0 & 67,433 aA & $100,000 \mathrm{cA}$ & $100,000 \mathrm{cA}$ & 90,000 bcA & 88,333 bA \\
\hline 0,8 & 92,267 a $B$ & 98,400 a $A$ & 99,667 a $A$ & 100,000 a $A$ & 100,000 a $\mathbf{B}$ \\
\hline 1,6 & 100,000 a B & 100,000 a $A$ & 100,000 a $A$ & 100,000 a $\quad A$ & $100,000 \mathrm{a}$ \\
\hline 2,4 & 96,133 a $B$ & 98,400 a $A$ & 96,133 a $A$ & 100,000 a $\mathrm{A}$ & $100,000 \mathrm{a}$ \\
\hline
\end{tabular}

(1) As médias seguidas de mesma letra minúscula, nas linhas, e maiú sculas, nas colunas, não diferem estatisticamente entre si, pelo teste Tukey, ao nível de 5\% de probabilidade.

Para todas as variáveis o fatorial vs testemunha relativa mostrou diferenças significati vas. No entanto, ocorreu o que já foi explicado para o rendimento de algodão em rama, ou seja algun s tratamentos herbicidicos não diferiram do contro- le mecânico, para todos as variáveis avaliadas.

O tratamento mecânico teve os seguin tes valores médi os: 40,67, 5080,06 $\mathrm{cm}^{2}$ e 2,54, respectivamente para número de folhas, área foliar por planta e LAI. 
Quadro 6 - Médias da lotação de plantas, na colheita, em percentagem da população máxima, em função das doses de diuron (kg/ha) e sethoxydim (g/ha). Ensaio envolvendo doses de diuron e sethoxydim. Viçosa, Minas Gerais, 1982 (1).

\begin{tabular}{|c|c|c|c|c|c|c|c|c|}
\hline \multirow{2}{*}{\multicolumn{2}{|c|}{$\begin{array}{l}\text { DOSES DO } \\
\text { DIURON }\end{array}$}} & \multicolumn{7}{|c|}{ DOSES DE SETHOXYDIM } \\
\hline & & \multirow{2}{*}{$\begin{array}{c}0,0 \\
59,600 \mathrm{aA}\end{array}$} & 150,0 & 300,0 & \multicolumn{2}{|l|}{450,0} & \multicolumn{2}{|l|}{600,0} \\
\hline gers & 0,0 & & $94,533 \mathrm{bc}$ & $100,000 \mathrm{cA}$ & $83,200 \mathrm{~b}$ & & 81,667 b & \\
\hline 9i:s. & 0,8 & 92,267 a $\mathrm{B}$ & 96,800 a & 98,067 a $A$ & 94,500 a & AB & $100,000 \mathrm{a}$ & B \\
\hline & 1,6 & 100,000 a B & $100,000 \mathrm{a}$ & 100,000 a $A$ & $100,000 \mathrm{a}$ & $\mathbf{B}$ & $100,000 \mathrm{a}$ & $\mathbf{B}$ \\
\hline & 2,4 & 96,133 a $\mathbf{B}$ & $98,400 \mathrm{a}$ & 95,133 a $A$ & $100,000 \mathrm{a}$ & B & $100,000 \mathrm{a}$ & $\mathbf{B}$ \\
\hline
\end{tabular}

(1) As médias seguidas de mesma letra minúscula, nas linhas, e maiú sculas, nas colunas, não diferem estatisticamente entre si, pelo teste Tukey, ao nível de 5\% de probabilidade.

Quadro 7 - Resumo das análises de variância dos dados de número de folhas por planta (NT), área foliar por planta, em cm2, índice de área foliar (LAI), aos 90 dias de plantio, em função dos tratamentos herbicídicos (fat orial) e o mecânico (teste mu nha relativa). Viçosa, Minas Gerais, 1982.

\begin{tabular}{|c|c|c|c|c|}
\hline \multirow{2}{*}{ FONTE DE VARIAÇAO } & \multirow{2}{*}{ GRAU DE LIBERDADE } & \multicolumn{3}{|c|}{ QUADRO MEDIO } \\
\hline & & NT & Area Foliar & LAI \\
\hline Blocos & 2 & $438,873^{* *}$ & $9367816,000^{* *}$ & $2,319^{* *}$ \\
\hline Fatorial & (19) & $234,670^{\star \star}$ & $2677633,000^{* *}$ & $0,768^{\star \star}$ \\
\hline Diuron (D) & 3 & $1269,711 * *$ & $17164304,000^{* \star}$ & 4,276 ** \\
\hline Sethoxydim (S) & 4 & 58,267 & 932907,750 & 0,226 \\
\hline D X S & 12 & 34,711 & 259477,500 & 0,072 \\
\hline Fatorial vs Test. Relativa & 1 & $1586,822^{\star \star}$ & $44021053,000^{* *}$ & $9,211^{* *}$ \\
\hline Tratamentos & 20 & $302,278^{k t k}$ & $4744804,000^{* *}$ & $1,191^{* *}$ \\
\hline Resíduo & 40 & 32,189 & 740396,00 & 0,186 \\
\hline CV (\%) & & 31,13 & 51,42 & 51,96 \\
\hline
\end{tabular}

* Significativo ao nível de $1 \%$ de probabilidade pelo teste F.

k A competição pro moveu hip otrofia foliar e redução no número de fol has Por outro lado, quando o processo emu lativo foi eliminado ou minimizado, devi do ao controle das plantas daninhas pelos herbicidas, os valo res obtidos para área foliar e LAI foram semelhantes ao: verificados em outros trabalhos $(20,23$ em especial para o diuron (quadro 8) dado o controle das latifoliadas que eram em maior densidade populacional no agroecossistema.

É necessário salientar que durante todo o período da cultura não houve fal- ta de umidade no solo, de modo que, embora seja difícil separar a competição por fatores do meio de maneira isolada (16), e que o solo foi adubado, a competição deve ter sido mai or por luz e pos sivelmente também por dióxido de carbono, pois o algodoeiro apresenta metabolismo fotossintético $\mathrm{C} 3$, com elevada taxa de fotorespiração (38).

Nenhum dos herbicidas mostrourse fitotóxico ao algodoeiro.

Com relação ao controle de plantas daninhas, as avaliações de densidade po- 
pulacional e peso da fitomassa hidratada epígea das espécies daninhas dominantes e do total delas, aos 60 dias do plantio, revelar am que o diuron control ou a maioria das latifoliadas, exceto a E. sonchifolia, e o sethoxydim con trolou totalmente a $B$. plantaginea. Houve interação entre os herbicidas no que diz respeito ao controle da gramínea em foco, para densidade populacional.
No quadro 9 tem-se as médias do número de espécimes de $B$. plantaginea por metro quadrado de superfície edáfica. Observa-se que o diuron, na ausência do sethox ydim, reduziu o número de indivíduos, porém não conseguiu um controle que possa ser considerado born. $\mathrm{O}$ sethoxydim por outro lado, mesmo na dose menor (150 g/ha) exerceu um excelente controle.

Quadro 8 - Médias do número de trofófilos por planta da (NT), área foliar por planta, em $\mathrm{cm} 2$, do índice de área foliar (LAI) aos 90 dias do plantio, em função das doses de diuron e sethoxydim. Viçosa, Minas Gerais, 1982 (1).

\begin{tabular}{cccc} 
Doses de diuron & \multicolumn{4}{c}{ Médias } \\
\cline { 2 - 4 } & $\mathrm{NT}$ & Area Foliar & LAI \\
\hline 0,0 & $5,33 \mathrm{~A}$ & $225,604 \mathrm{~A}$ & $0,111 \mathrm{~A}$ \\
0,8 & $14,867 \mathrm{~B}$ & $1185,254 \mathrm{~B}$ & $0,591 \mathrm{~B}$ \\
1,6 & $21,600 \mathrm{C}$ & $1838,136 \mathrm{~B}$ & $0,892 \mathrm{~B}$ \\
2,4 & $26,600 \mathrm{C}$ & $2763,102 \mathrm{C}$ & $1,384 \mathrm{C}$
\end{tabular}

(1) Em cada coluna, médias seguidas pela mes ma letra, não diferem entre si, pelo teste Tukey, ao nível de $5 \%$ de probabilidade.

Quadro 9 - Médias do número de espécies da Brachiaria plantaginea por $\mathrm{m}^{2}, 60$ dias do plantio, dados transformados para $\quad \mathbf{x + 1}$,em função das doses de diuron $(\mathrm{kg} / \mathrm{ha})$ e sethoxydim (g/ha). Ensaio envolvendo doses de diuron e sethoxydim. Viçosa, Minas Ge. rais. $1981(1)$.

\begin{tabular}{cccccc}
\hline Doses do Diuron & \multicolumn{5}{c}{ Doses de Sethoxydim } \\
\cline { 2 - 6 } & 0,0 & 150,0 & 300,0 & 450,0 & 600,0 \\
\hline 0,0 & $8,519 \mathrm{aA}$ & $1,000 \mathrm{bA}$ & $1,000 \mathrm{bA}$ & $1,000 \mathrm{bA}$ & $1,000 \mathrm{bA}$ \\
0,8 & $6,675 \mathrm{a}$ B & $1,412 \mathrm{bA}$ & $1,000 \mathrm{bA}$ & $1,000 \mathrm{bA}$ & $1,000 \mathrm{bA}$ \\
1,6 & $5,675 \mathrm{a} \mathrm{BC}$ & $1,000 \mathrm{bA}$ & $1,000 \mathrm{bA}$ & $1,000 \mathrm{bA}$ & $1,000 \mathrm{bA}$ \\
2,4 & $5,161 \mathrm{a} \quad \mathrm{C}$ & $1,667 \mathrm{bA}$ & $1,000 \mathrm{bA}$ & $1,000 \mathrm{bA}$ & $1,000 \mathrm{bA}$ \\
\hline \hline
\end{tabular}

(1) As médias seguidas de mesma letra minúscula, nas linhas, e maiúscula, nas colunas, não diferem estatisticamente entre si, pelo teste Tukey, ao nível de 5\% de probabilidade.

A redução da fitomas sa hidratada epíge a da $B$. plantaginea foi notável, quando aplicou-se o sethoxydim (quadro 10). Neste caso, o diuron não teve efeito significativo, o que vem a demonstrar que na avaliação do efeito herbicida é necessário o uso de dois ou mais métodos sinecológicos, pois apenas a contagem de in- divíduos não é suficiente para comprovar o grau de controle de um determinado produto.

É necess ário salientar que, quando da aplicação do sethoxydim, a supra referida gramínea estava com uma alt ura média de $25 \mathrm{~cm}$, e uma considerável área foliar. 
Quadro 10 - Médias em g, da fitomassa hidra tada epígea de Brachiaria plantaginea por $\mathrm{m}^{2}, 60$ dias do plantio, dados transformados para Log $(x+1)$, em função das doses de sethoxydim (g/ha). Ensaio en volvendo doses de diuron e se thoxydim. Viçosa, Minas Gerais, 1981 (1)

\begin{tabular}{|c|c|}
\hline Doses de Sethoxydim & Médias \\
\hline $\begin{array}{r}0,0 \\
150,0 \\
300,0 \\
450,0 \\
\quad 600,0\end{array}$ & $\begin{array}{ll}2,510 & \mathrm{~A} \\
\mathbf{0 , 1 5 1} & \mathrm{B} \\
0,000 & \mathrm{~B} \\
0,000 & \mathrm{~B} \\
0,000 & \mathrm{~B}\end{array}$ \\
\hline
\end{tabular}

Na coluna, médias seguidas pela mesma letra, não diferem entre si, pelo teste Tukey, ao nível de $5 \%$ de probabilidade.

As médias de número de espécimes e da fitomassa hidratada epígea, 60 dias após o plantio da cultura, por metro quadrado de solo de G. parviflora, E. sonchifolia e do total de todas as espécies encontram se no quadro 11, consider and o o fat or "doses de diuron", significativo pelo teste F na análise de variância.

Verifica-se que por não ter sido controlada por nenhum dos dois herbicidas, a E. sonchifolia mostrou sensíveis aumentos da fitomassa hidratada epígea e densidade populacional, embora não significativos, à medida que se aumentou a dose do diuron. $\mathrm{Na}$ verdade, é um efeito indireto, pois sendo as demais espécies sensíveis ao diuron e sethoxydim, neste caso a $B$. plantaginea, a $E$. sonchifolia ficou livre da pressão de competição das demais espécies, e assim teve oportunidade de crescer mais (quadro 11). No entanto, mostrou ser uma espécie de baixa capacidade competitiva com o algodoeiro, pois os atributos do crescimento e desenvolvimento dessa malvácea não sofreram reduções significativas.

Quadro 11 - Médias do número de espécies de Galinsoga parviflora (GP), Emilia sonchifolia (ES), e total de todas as espécies (TS), por $\mathrm{m}^{2}$, dados transformados para $\mathrm{v} x+1$, fitomassa hidratada epígea (FHE) de GP, ES e TS, em g. ( $\left.{ }^{2}\right){ }^{1}{ }^{1}$, transformados para $\operatorname{Lg}(x+1), 60$ dias do plantio, em função dos doses de diuron $(\mathrm{kg} / \mathrm{ha})$. Ensaio envolvendo doses de diuron e sethodydim. Viçosa, Minas Gerais, 1982 (1).

\begin{tabular}{|c|c|c|c|c|c|c|}
\hline \multirow{3}{*}{ Doses de Diuron } & \multicolumn{6}{|c|}{ Médias } \\
\hline & \multicolumn{3}{|c|}{ Contagem } & \multicolumn{3}{|c|}{ FHE } \\
\hline & GP & ES & TS & GP & ES & TS \\
\hline 0,0 & $13,222 \mathrm{~A}$ & $6,407 \mathrm{~A}$ & $16,839 \mathrm{~A}$ & $2,392 \mathrm{~A}$ & 2,579 В & $3,158 \mathrm{~A}$ \\
\hline 0,8 & $3,511 \quad \mathrm{~B}$ & $7,135 \mathrm{~A}$ & $10,073 \quad$ В & $1,189 \quad \mathrm{~B}$ & $3,145 \mathrm{~A}$ & $3,340 \mathrm{~A}$ \\
\hline 1,6 & $1,472 \mathrm{~B}$ & $7,570 \mathrm{~A}$ & 8,406 B & $0,420 \quad \mathrm{BC}$ & $3,327 \mathrm{~A}$ & $3,321 \mathrm{~A}$ \\
\hline 2,4 & $1,291 \quad \mathrm{~B}$ & $6,577 \mathrm{~A}$ & 7,295 B & $0,202 \quad \mathrm{C}$ & $3,140 \mathrm{~A}$ & $3,179 \mathrm{~A}$ \\
\hline
\end{tabular}

Em cada coluna, médias seguidas pela mesma letra, não diferem entre si, pelo teste Tukey, ao nível de $5 \%$ de probabilidade.

Considerando o total de indivídu os de todas as espécies, verificou-se que o diuron, mesmo na dose mais baix a $(0,8$ $\mathrm{kg} /$ ha 1 , já reduziu significativamente tal variável. Isto devido, ao fato de ser a G. parviflora, a espécie domi nante em densidade populacional, cont rola da pelo produto ( quadro 11). No entanto, quando se considera a fitomassa hidratada epígea, não houve contrastes significativos entre doses do diuron. Neste caso, face a redução da força competitiva global, devido ao controle das demais es pécies, a $E$. sonchifolia, que na ausência do diuron apresentava esta variável reduzida, cresceu mais, embora apresentasse baixa capacidade competitiva com a cultura.

Este fato mostra que a simples avaliação da biomassa de todas as espécies 
daninhas de um determinado agroecossis tema, não é um índice con fiả vel do aspecto emulativo e do controle de plantas dan inhas. Pelo contrário, às vezes pode ocorrer a cooperação mútua, em vez da competição, ou também o neutralismo competitivo. Outro fator de importância é que a competitividade varia com a espécie daninha, o que aliás já foi observado anteriormente $(9,10,32)$.
Os graus de associação, através dos coeficientes de correlação de Pearson, entre as den sidades populacionais das plantas daninhas dominantes, e o total de todas as espécies, bem como entre a fitomassa hidratada epigea por espécie de planta daninha e o total delas com o atributo do crescimento e desenvolvimento do algodoeiro, estão contidos nos quadros 12 e 13 .

Quadro 12 - Graus de associação, através dos coeficientes de correlação de Pears on, entre núme ro de espécimes por $\mathrm{m}^{2}$, aos 60 dias do plantio, do total de todas as espécies (Y1), de Galinsoga parviflora $_{(\mathrm{Y} 2),}$ de Brachiaria plantaginea ${ }_{(\mathrm{Y} 3)}$ e de Emilia sonchifolia (Y4) e da fitomassa hidratada epigea em g. $\left(\mathrm{m}^{2}\right)_{-}{ }^{1}$, aos 60 dias do plantio, do total de todas as espécies (Y5), de Galinsoga parviflora (Y6), de Brachiaria plantaginea (Y7) e de Emilia sonchifolia (Y8), com rendimento de algodão em rama, em kg/ha (Y15), altura média plantular, na colheita, em $\mathrm{cm}$ (Y16), altura média plantular, aos 90 dias do plantio, em $\mathrm{cm}$ (Y17), diâmetro caulinar, na colheita, em $\mathrm{cm}$ (Y18), diâmetro caulinar, aos 90 dias do plantio, em $\mathrm{cm}$ (Y 19), número de ramos vegetativos por planta, na colheita (Y20), lotação de plantas, na colheita, em percentagem (Y21) e lotação de plantas, após desbaste, em percentagem (Y22). Ensaio envolvendo doses de diuron e sethoxydim. Viçosa, Minas Gerais, 1982.

\begin{tabular}{ccccccccr}
\hline Variáveis & Y1 & Y2 & Y3 & Y4 & Y5 & Y6 & Y7 & Y8 \\
\hline Y15 & $-0,58^{*}$ & $-0,51^{\mathrm{ns}}$ & $-0,27^{\mathrm{ns}}$ & $0,04^{\mathrm{ns}}$ & $-0,12^{\mathrm{ns}}$ & $-0,48^{*}$ & $-0,23^{\mathrm{ns}}$ & $0,26^{\mathrm{ns}}$ \\
Y16 & $-0,42^{\star}$ & $-0,33^{\mathrm{ns}}$ & $-0,11^{\mathrm{ns}}$ & $-0,12^{\mathrm{ns}}$ & $-0,21^{\mathrm{ns}}$ & $-0,30^{\mathrm{ns}}$ & $-0,01^{\mathrm{ns}}$ & $-0,02^{\mathrm{ns}}$ \\
Y17 & $-0,62^{*}$ & $-0,53^{*}$ & $-0,20^{\mathrm{ns}}$ & $-0,02^{\mathrm{ns}}$ & $-0,11^{\mathrm{ns}}$ & $-0,54^{*}$ & $-0,12^{\mathrm{ns}}$ & $0,23^{\mathrm{ns}}$ \\
Y18 & $-0,52^{\star}$ & $-0,44^{*}$ & $-0,14^{\mathrm{ns}}$ & $-0,02^{\mathrm{ns}}$ & $-0,09^{\mathrm{ns}}$ & $-0,39^{*}$ & $-0,03^{\mathrm{ns}}$ & $0,13^{\mathrm{ns}}$ \\
Y19 & $-0,58^{*}$ & $-0,50^{*}$ & $-0,23^{\mathrm{ns}}$ & $0,06^{\mathrm{ns}}$ & $-0,11^{\mathrm{ns}}$ & $-0,52^{*}$ & $-0,13^{\mathrm{ns}}$ & $0,22^{\mathrm{ns}}$ \\
Y20 & $-0,38^{\mathrm{ns}}$ & $-0,31^{\mathrm{ns}}$ & $-0,18^{\mathrm{ns}}$ & $0,01^{\mathrm{ns}}$ & $0,08^{\mathrm{ns}}$ & $-0,32^{\mathrm{ns}}$ & $-0,19^{\mathrm{ns}}$ & $0,22^{\mathrm{ns}}$ \\
Y21 & $-0,33^{\mathrm{ns}}$ & $-0,26^{\mathrm{ns}}$ & $-0,53^{*}$ & $0,16^{\mathrm{ns}}$ & $0,01^{\mathrm{ns}}$ & $-0,30^{\mathrm{ns}}$ & $-0,44^{*}$ & $0,26^{\mathrm{ns}}$ \\
Y22 & $-0,37^{\mathrm{ns}}$ & $-0,29 \mathrm{~ns}$ & $-0,66^{*}$ & $0,13^{\mathrm{ns}}$ & $-0,02^{\mathrm{ns}}$ & $-0,34^{\mathrm{ns}}$ & $-0,54^{*}$ & $0,25^{\mathrm{ns}}$
\end{tabular}

ns: Não significativo ao nível de $1 \%$ de probabilidade pelo teste $t$. Significativo ao nível de $1 \%$ de probabilidade pelo teste t.

Verifica-se que todas as correlações signific ativas, foram negativas, indicando as sim, um relacionamento inverso entre o cres cime nto do complexo flor istico daninho e a cul tura, à sem elh ança do que foi verificado por Savage \& Bardsley (34).

O "strain" biológico causado pela competição (26 ), o qual resulta do somatório de diversos "strains", tais como competição por luz, onde cada folha comporta-se como sendo unidades indivi- duais (18), por $\mathrm{CO} 2$, água, nutrientes e aspectos alelopáticos, leva a cultura a sofre r transform ações plásticas irr eversí veis, o que culmina com a redução do crescimento como um todo.

Observando-se os quadros 12 e 13 notase que a $G$. parviflora foi a planta que ocasionou valores absolutos maiores nos coeficientes de correlação, indicando que, devido a sua maior densidade populacional ela reduziu mais os atributos do crescimento da cultura do que as demais. Por 
Quadro 13 - Graus de associação, através dos coeficientes de correlação de Pearson, entre núme ro de espécimes por $\mathrm{m}^{2}$, aos 60 dias do plantio, do total de todas as espécies (Y1), de Galinsoga parviflora (Y2), de Brachiaria plantaginea (Y3) e de Emilia sonchifolia (Y4), e de fitomassa hidratada epígea por $\mathrm{m}^{2}$, aos 60 dias do plantio, do total de todas as espécies (Y5), de Galinsoga parviflora (Y6), de Brachiaria plantaginea (Y7) e de Emilia sonchifolia (Y8) com número de trofófilos por planta, aos 90 dias do plantio (Y9), área foliar por planta, aos 90 dias do plantio, em $\mathrm{m}_{(\mathrm{Y} 10)}$ e índice de área foliar aos 90 dias do p lantio (Y 11), referentes ao al godoeiro. Ensaio envolvendo doses de diuron e sethoxydim. Viçosa, Minas Gerais, 1982.

\begin{tabular}{ccccccccc} 
Variáveis & Y1 & Y2 & Y3 & Y4 & Y5 & Y6 & Y7 & Y8 \\
\hline Y9 & $-0,53^{*}$ & $-0,45^{*}$ & $-0,22^{\text {ns }}$ & $-0,02^{\text {ns }}$ & $-0,12^{\text {ns }}$ & $-0,45^{*}$ & $-0,11^{\mathrm{ns}}$ & $0,17^{\mathrm{ns}}$ \\
Y10 & $-0,47^{*}$ & $-0,40^{*}$ & $-0,15^{\mathrm{ns}}$ & $-0,06^{\mathrm{ns}}$ & $-0,21^{\mathrm{ns}}$ & $-0,39^{*}$ & $-0,07^{\mathrm{ns}}$ & $0,06^{\mathrm{ns}}$ \\
Y11 & $-0,47^{*}$ & $-0,39^{*}$ & $-0,14^{\mathrm{ns}}$ & $-0,10^{\mathrm{ns}}$ & $-0,22^{\mathrm{ns}}$ & $-0,39^{*}$ & $-0,07^{\mathrm{ns}}$ & $0,05^{\mathrm{ns}}$
\end{tabular}

${ }^{1 s}$ : Não significativo ao nível de $1 \%$ de probabilidade pelo teste t. *: Significativo ao nível de $1 \%$ de probabilidade pelo teste $\mathrm{t}$.

Quadro 14 - Graus de associação entre número de espécimes de todas as espécies (Y1), de Galinsoga parviflora (Y2), de Brachiaria plantaginea (Y3), de Emilia sonchifolia (Y4), por $\mathrm{m}^{2}$, e fitomassa hidratada epígea de todas as espécies (Y5), de Galinsoga parviflora (Y6), de Brachiaria plantaginea (Y7) e de Emilia sonchifolia (Y8), em g. $\left(\mathrm{m}^{2}\right){ }^{1}$, aos 60 diasdo plantio. Ensaio envolvendo doses de diuron e sethoxydim. Viçosa, Minas Gerais, 1982.

\begin{tabular}{ccccccccc}
\hline Variáveis & Y1 & Y2 & Y3 & Y4 & Y5 & Y6 & Y8 & Y7 \\
\hline Y1 & - & $0,95^{*}$ & $0,30^{\mathrm{ns}}$ & $0,04 \mathrm{~ns}$ & $-0,10^{\mathrm{ns}}$ & $0,81^{*}$ & $0,61^{\mathrm{ns}}$ & $-0,40^{*}$ \\
Y2 & - & - & $0,16^{\mathrm{ns}}$ & $-0,15 \mathrm{~ns}$ & $-0,25^{\mathrm{ns}}$ & $0,81^{*}$ & $0,04 \mathrm{~ns}$ & $-0,45^{*}$ \\
Y3 & - & - & - & $-0,07^{\mathrm{ns}}$ & $-0,09 \mathrm{~ns}$ & $0,28^{\mathrm{ns}}$ & $0,86^{*}$ & $-0,23^{\mathrm{ns}}$ \\
Y4 & - & - & - & - & $0,43^{*}$ & $-0,12^{\mathrm{ns}}$ & $-0,14^{\mathrm{ns}}$ & $0,45^{*}$ \\
Y5 & - & - & - & - & - & $-0,14^{\mathrm{ns}}$ & $0,09 \mathrm{~ns}$ & $0,68^{*}$ \\
Y6 & - & - & - & - & - & - & $0,13 \mathrm{~ns}$ & $-0,43^{*}$ \\
Y7 & - & - & - & - & - & - & - & $-0,22^{\mathrm{ns}}$ \\
Y8 & - & - & - & - & - & - & - & -
\end{tabular}

ns: Não significativo ao nível de $1 \%$ de probabilidade pelo teste t.

*: Significativo ao nível de $1 \%$ de probabilidade pelo teste $t$.

outro lado, a B. plantaginea, apesar de ser uma planta agressiva, por ter menor densidade populacional, não se correlacio nou com as características do crescimento da cultura.

A E. sonchifolia, apesar de ter produzido uma fitoma ssa con siderável, não afe tou o cre scimen to do alg odo eiro, o que foi refletido pelos baixos e insignificantes coeficientes de correlação obtidos.
O elevado coeficiente de correlação obtido entre o número de espécies de $G$. parviflora e o total de todas as espécies $(\mathrm{r}=0,95)$, e valore s não sig nificativos para as de mais es pécies, mostra que aquela asterácea era dominante do complex o flor istico, pelo menos em termos de densidade populacional ou abundância (quadro 14).

No entanto, considerando-se a fito- 
mass a formada pela G. parviflora e o total de todas as espécies, verificou-se ausência de correlação, o que mostra que ela não foi a espécie mais agressiva do complexo, pois quando não controlada, assim como as demais, os indivíduos eram raquíticos e estiolados, indicando que as demais espécies daninhas, em especial a $B$. plantaginea, exerceram e ganharam na competição, pelo menos, pela luz.

A correlação significativa entre a fitomassa total de todos os indivíduos e a da $E$. sonchifolia, de valor positivo, mostra que apesar de ter um menor número de espécimes, a referida planta cresceu normalmente, pois não foi controlada pelos herbicidas testados.

\section{LITERATURA GITADA}

1. Alves, A. \& Forster, R. Variaçōes nos métodos de aplicação dos herbicidas diuron e trifluralin na cultura do algodoeiro. Bragantia 26: 253-264, 1967.

2. Ashely, D.A.; Doss, B.D. \& Bennet, O.L. A method of determining leaf area in cotton. Agron. J. 55: 584-585, 1963.

3. Beltrão, N.E. de M.; Canuto, V.T.B. \& Aguiar, M.J.N. Influência competitiva da tiririca e do capim-de-burro sobre o algodoeiro herbáceo, cultivar AFC 38-12. Pesq. Agropec. Bras, 13: 35-43, 1978.

4. Beltrão, N.E. de M.; Azevedo, D.M.P. \& Lima, R.N. Interaçăo entre os efeitos da competição de plantas daninhas, da adubação nitrogenada e da cultivar em algodoeiro herbáceo (Gossypium hirsutum latifolium L.). Campina Grande, Paraíba. Centro Nacional de Pesquisa do Algodão - EMBRAPA. 1979. p. 1-25. (Boletim Técnico, 1)

5. Beltrāo, N.E. de M.; Azevedo, D.M.P. \& Lima, R.N. Competição entre plantas daninhas e o algodoeiro herbáceo "Gossypium hirsutum latifolim L." nos Estados da Paraiba e Pernambuco. Campina Grande, Pa. raíba. Centro Nacional de Pesquisa do $\mathrm{Al}$ -

- godäo - EMBRAPA. 1979. p. 1-22. (Boletim Técnico, 2).

6. Brandão, M.; Laca-Buendia, J.P.C. \& Gavilanes, M.L. Principais plantas daninhas no Estado de Minas Gerais. Inf. Agropec. 8: 18-26, 1982.

7. Braun Blanquet, J. Fitosociologia. Bases para el estudio de las comunidades vegetales. Traducido por Lalucat Jo, J. Madrid, España. H. Blume Ediciones. 1979. 820 p.
8. Buchanan, G.A. \& Burns, E.R. Influence of weed competition. Weeds Sci. 18: 149-154, 1970.

9. Buchanan, G.A. \& Burns, E.R. Weed compe. tition in Cotton. I. Sicklepond and tall mornigglory. Weed Sci. 19: 576-579, 1971.

10. Buchanan, G.A. \& Burns, E.R. Weed competition in cotton. II. Cocklebur and redroot piweed. Weed Sci. 19: 580-582, 1971.

11. Calzada Benza, J. Métodos Estatisticos para la investigaciou. 2." ed. Lima, Peru. 1964. p. 381-386.

12. Cardenas, J,; Reyes, C.E. \& Doll, J.D. Tropicales. Weeds. Malezas Tropicales. Vol. 1. Bogotá, Colombia. Instituto Colombiano Agropecuário. 1972. 341 p.

13. Church, J.M.F. Developments in weed contral in Tanzania and Uganda Cotton Grow. Rev. 49: 341-349, 1972.

14. Cruz, L.S.P. \& Leiderman, L. Aplicação de misturas de diuron com MSMA e com paraquat, no controle de plantas daninhas de folhas largas em cultura de algodão (Gossypium hirsutum L.). Planta Daninha 1: 45-50, 1978.

15. Cruz, L.S.P. \& Borgo, A. Emprego de misturas de herbicidas nas entrelinhas de algodoeiros para o controle de plantas daninhas tardias. In: Congresso Brasileiro de Herbicidas e Ervas Daninhąs, XIII, Ilhéus, 1980. Resumos, p. 3.

16. Donald, C.M. Competition among crop and pasture plants. Advan. Agron. 15: 1-118, 1963.

17. Dowler, C.C. \& Houser, E.W. Weed control systems in cotton on tifton loamy sand soil. Weed Sci. 23: 40-42, 1975.

18. Etherington, J.R. Competition. In: Environment and plant ecology John Willey \& Sons. 1975. p. 276-308.

19. Furtick, W.R. \& Romanowski, R.R. Manual de métodos de investigacion de maleza. México. Centro Nacional de Qyuda Técnica. 1971.82 p.

20. Hearn, A.B. Crop physiology. In: Arnold, M. H. (ed.) Agricultural research for devetopment. The Namulonge contribution. Cotton Research Corporation. Cambridge, Cambridge University Press. 1973. p. 77 . 122.

21. Hunt, R. Plant growth analysis. The Institute of Biology's. London. Studies in Biology n. 96. Edward Arnold. 1978. 67 p.

22. Laca-Buendia, J.P.C.; Purcino, A.A.C.; Penna, J.C.V. \& Ferreira, L. Período crítico de 
competiçāo entre comunidades de plantas daninhas e o algodoeiro (Gossypium hir. sutum L.) no Estado de Minas Gerais. Planta Daninha 2: 89-95, 1979.

23. Karami, E. \& Weaver Jr. J.B. Growth analysis of american upland cotton, Gossypium hirsutum L., with different leaf shapes and colors. Crop Sci. 12: 317-320, 1972.

24. Kerr, H.D. Herbicides systems of cotton. Proc. South weed Sci. Soc. 24: 85-86, 1971.

25. La Loma, J.L. Experimentacion agricola. México. Union Tipográfica Editorial Americana. $1955.430 \mathrm{p}$.

26. Levitt, $J$. the nature of stress injury and resistance. In: Respounes of plants to environmental stress. New York, U.S.A. Academic Press. 1972. p. 9-16.

27. Leiderman, L.; Santos, C.A.L.; Figueiredo, P. \& Silveira, R.I. Controle de ervas daninhas do algodão com misturas de trifluralin e diuron em quatro regiōes de São Paulo. $O$ Biológico 32: 158-163, 1966.

28. Moran-Val, C.A. \& Miller, P.A. Inter-riw competitive effects among four cotton cultivars. Crop Sci. 15: 479-482, 1975.

29. Pimentel Gomes, F. Curso de Estatistica Experimental. 4.: edição. Piracicaba, Săo Paulo. Livraria Nobel S.A. 1970. 430 p.
30. Righi, N.R.; Ferraz, C.A.M. \& Corrêa, D.M. Cultural. In: Neves, O.S. et al. Cutura e adubação do algodoeiro. São Paulo, Instituto Brasileiro de Potassa. 1965. p. 255-317.

31. Robinson, E.L. Yield and height of cotton as affected by weed density and nitrogen level. Weed Sci. 24: 40-42, 1976.

32. Robinson, E.L. Effect of weed species and placement on seed cotton yield. Weed Sci. 24: 353-355, 1976.

33. Rogers, N.K.; Buchanan, G.A. \& Johnson, W. C. Influence of row spacing ou weed com petition with cotton. Weed Sci. 24: 410-413, 1976.

34. Savage, K.E. \& Bardsley, C.E. Relation of lime and preemergence herbicides to yields of cotton and weeds. Agron. J. 58: 269-271, 1966.

35. Schwerzel, P.J. \& Thomas, P.E.L. weed competition in cotton Pans 17: 30134, 1971.

36. Tollervey, F.E. weed control in cotton. Cot. ton Grow. Rev. 51: 247-255, 1974.

37. Weed Science Society of america. Herbicide Handbook. Illinois, U.S.A. Fouth Edition. 1979. p. $188-193$.

38. Zelitch, I. Photosynthesis, Photorespiration and plan tproductivity. New York, U.S.A Academic Press. 1971. 337 p. 